\title{
Guidelines for Submitting Adverse Event Reports for Publication
}

\author{
William N. Kelly, ${ }^{1}$ Felix M. Arellano, ${ }^{2,3}$ Joanne Barnes, ${ }^{4}$ Ulf Bergman, ${ }^{5}$
}

Ralph I. Edwards, ${ }^{6}$ Alina M. Fernandez, ${ }^{7}$ Stephen B. Freedman, ${ }^{8}$ David I. Goldsmith, ${ }^{9}$

Kui A. Huang, ${ }^{10}$ Judith K. Jones, ${ }^{11}$ Rachel McLeay, ${ }^{12}$ Nicholas Moore, ${ }^{13}$

Rosie H. Stather, ${ }^{12}$ Thierry Trenque, ${ }^{14}$ William G. Troutman, ${ }^{15}$ Eugene van

Puijenbroek, ${ }^{16}$ Frank Williams ${ }^{17}$ and Robert P. Wise ${ }^{18}$

1 William N. Kelly Consulting, Inc., Oldsmar, Florida, USA

2 Risk Management Resources, Bridgewater, New Jersey, USA

3 Risk Management Resources, Zaragoza, Spain

4 University of Auckland, Auckland, New Zealand

5 Karolinska Institute, Stockholm, Sweden

6 Uppsala Monitoring Centre, Uppsala, Sweden

7 TAP Pharmaceutical Products, Inc., Lake Forest, Illinois, USA

8 The Hospital for Sick Children, Toronto, Ontario, Canada

9 Goldsmith Pharmacovigilance and Systems, New York, New York, USA

10 Pfizer Pharmaceuticals, New York, New York, USA

11 The Degge Group, Ltd., Arlington, Virginia, USA

12 Wolters Kluwer Health, Auckland, New Zealand

13 Université Victor Segalen, Bordeaux, France

14 Centre Hospitalier Universitaire, Reims, France

15 University of New Mexico, Albuquerque, New Mexico, USA

16 Netherlands Pharmacovigilance Centre, 's-Hertogenbosch, The Netherlands

17 United States Navy, Bethesda, Maryland, USA

18 United States Food and Drug Administration, Rockville, Maryland, USA

\section{Abstract}

Publication of case reports describing suspected adverse effects of drugs and medical products that include herbal and complementary medicines, vaccines, and other biologicals and devices is important for postmarketing surveillance. Publication lends credence to important signals raised in these adverse event reports. Unfortunately, deficiencies in vital information in published cases can often limit the value of such reports by failing to provide enough details for either (i) a differential diagnosis or provisional assessment of cause-effect association, or (ii) a reasonable pharmacological or biological explanation. Properly described, a published report of one or more adverse events can provide a useful signal of possible risks associated with the use of a drug or medical product which might warrant further exploration. A review conducted by the Task Force authors found that many major journals have minimal requirements for publishing adverse event reports, and some have none at all. Based on a literature review and our collective experience in reviewing adverse event case reports in regulatory, academic, and industry settings, we have identified information that we propose should always 
be considered for inclusion in a report submitted for publication. These guidelines have been endorsed by the International Society for Pharmacoepidemiology (ISPE) and the International Society of Pharmacovigilance (ISoP) and are freely available on the societies' websites. Their widespread distribution is encouraged. ISPE and ISoP urge biomedical journals to adopt these guidelines and apply them to case reports submitted for publication. They also encourage schools of medicine, pharmacy, and nursing to incorporate them into the relevant curricula that address the detection, evaluation, and reporting of suspected drug or other medical product adverse events.

\section{Introduction}

Publication of adverse event reports represents an important part of postmarketing safety surveillance of drugs and medical products that include herbal and complementary medicines, vaccines, and other biologicals and devices. Such reports help identify potential product-associated risks and serve as signals of possible events that may require more formal studies. When properly documented, reports of one or more adverse events can help to alert clinicians to these possible effects. More importantly, hypotheses can be developed on product-associated effects that can be formally evaluated and quantified in clinical or observational studies. This process ultimately gives decision makers a more complete understanding of a drug or medical product's potential for benefits and risks.

Adverse event case reports typically originate with healthcare providers who, while caring for patients, suspect a potentially causal relationship between a medical product and an adverse event. Besides reporting this case or a case series as requested or required by the relevant national health authorities, the healthcare provider or the health authority (for example, the US FDA) may also elect to submit the observations to a biomedical journal for publication.

Adverse event reports published in biomedical journals can have a significant clinical impact, especially for rare events that might not be detected in clinical trials. They can serve as signals of possible problems to increase awareness of the possible association and stimulate further reports. Readers might assume that these published reports have passed the intense scrutiny of the editorial and peer-review processes (whether this is true or not should be made clear by the journal). However, the completeness of published reports has been shown to vary. ${ }^{[1-13]}$ Their value is limited, and they can even be misleading when they lack relevant information regarding the patient, the event, all potentially relevant exposures, the clinical decision-making processes and, most importantly, the possible alternative aetiologies. Even when they contain all the essential information, it is often not possible to arrive at a definitive diagnosis for the event.

Several reports (a case series) might provide a stronger signal than a single case, although they may also simply reflect systematic confounding by indication or other biases. These reports build the foundation for explanatory hypotheses, but they cannot provide information on the quantitative population risk, because they represent an unknown proportion of the adverse events associated with a medical product (unknown numerator due to under-reporting), and the total number of exposed patients is not provided (unknown denominator). They also cannot provide balanced information on the risk factors for such an event. Identifying and quantifying risk factors require population-based or other types of epidemiological studies.

\section{The Need for Guidelines}

Past reviews and editorials have expressed concern about the quality of published adverse event reports written by practitioners. ${ }^{[1-13]}$ In 1985, an international conference attended by professionals interested in adverse drug events and by editors of 
several major medical journals proposed guidelines that editors should adopt when reviewing adverse event reports submitted for publication. ${ }^{[1]}$ Another working group commissioned by the French authorities also published recommendations in 1997. ${ }^{[14]}$ However, a recent review found that many major journals still have minimal requirements for publishing adverse event reports, and some have none at all. ${ }^{[12]}$

\section{Methods}

Because of the deficiencies of many published adverse event reports and inconsistent publication requirements, the International Society for Pharmacoepidemiology (ISPE) Board of Directors appointed a Task Force in 2004 to examine the need for guidelines on publishing adverse event reports. This Task Force was comprised of professionals from North America, Europe, and New Zealand with expertise in clinical pharmacology, pharmacoepidemiology, pharmacovigilance, drug regulation, medicine, pharmacy, herbal medicines, medication safety, and biomedical publishing.

The Task Force performed a review of the literature from 1966 to 2005 to identify any previous guidelines on publishing adverse event reports and to evaluate the need for new guidelines. Searches using the search terms 'adverse' and 'guidelines', and 'adverse' and 'published' were done using MEDLINE. Other relevant publications on causality assessment were solicited from experts in the field.

\section{Results}

The literature search disclosed a continuing concern about the quality of published adverse event reports. ${ }^{[2-13]}$ The review revealed only one usable set of guidelines published in 1985. ${ }^{[1]}$ Another set of guidelines published in 1997 was not readily accessible. ${ }^{[14]}$ The original 1985 guidelines built on the structure and data elements requested in regulatory adverse event reporting forms such as the US FDA's MedWatch Form 1639 (predecessor to the current MedWatch 3500 form) and the Council for International Organizations of Medical Sciences form used by many regulatory agencies worldwide. Further, the elements included in the European Single Case in Pharmacovigilance Exchange (EuroSCaPE) project (part of the European Union's Trade Electronic Data Interchange System) that evolved into the International Conference on Harmonisation (ICH) E2B standard elements for transmission of individual case safety reports were also considered to identify key elements in case reports. ${ }^{[15,16]}$

\section{The Guidelines}

The Task Force developed the following guidelines with broad participation from interested ISPE and International Society of Pharmacovigilance (ISoP) members, and the boards of directors of both organisations have approved them.

Table I provides the data elements that the Task Force recommends should be considered when constructing an adverse event report for publication. Many of the elements listed as 'required' for adverse event case reports are based upon the study of adverse event causality assessment by Hill ${ }^{[17]}$ and the validated work of Naranjo et al. ${ }^{[18]}$ Although some of the recommended elements might not be relevant in specific adverse event cases, such as medication errors or drug interactions, authors submitting reports for publication should provide explanations if any of the recommended information is missing from a report, in order to clarify whether the information was available but not reported or just not available. In addition, authors of adverse event case reports should have reported the case to the appropriate regulatory authority and, if possible, provide the report number to help identify duplicates that might also be included in reports submitted by the authority.

The adverse event case report should be enough to describe the features of the case that explain the above elements. Some case reports will necessarily be longer than others based on complexity. Thus, journal requirements to comply with a specific word count for an adverse event case report may hinder a proper description of the case and should be avoided. 
Consistent with the content of the report

\section{Patient}

Demographics

Current health status

Medical history

Physical examination

Patient disposition

\section{Identification}

\section{Dosage}

Administration

Drug-reaction interface

Concomitant therapies

\section{Age group, sex}

Disease or symptoms being treated with suspect drug

Medical history relevant to adverse event

Abnormal physical or laboratory findings.

For off-label use, documentation of the

reason

Presence or absence of death, life-

threatening circumstances, hospitalisation or prolonged hospitalisation or significant disability

Suspected drug identified by generic name. Herbal products can be described by Latin binomial of herbal ingredients, plants part(s), and type of preparation

(e.g. crude herb or extract). Proprietary name and name of producer for manufactured products

Approximate dosage, duration of therapy

Therapy duration before the adverse event

Assessment of potential contribution of concomitant therapies

\section{Exact age, weight}

Duration of illness

Prior exposure to drug product or class. Underlying risk factors

Baseline laboratory findings with norma range of values of the laboratory

Status several months after adverse event

\section{Suspected brand name with strength/}

dosage unit. For herbal extracts, type and concentration of extraction solvent used.

For herbal products, state whether or not

the product(s) implicated are authorised or licensed, and whether or not sample(s)

have been retained for analysis, and any results

Exact dosage, start and stop dates

Route

First dose-event interval, last dose-event interval

Description of concomitant therapies, including non-prescription, herbal or complementary medicines
Height, race and ethnicity, obstetrica status, body mass index, occupation Severity of disease/symptoms. Previous therapy of active disease

Alcohol, tobacco, and substance abuse history, relevant social circumstances,

family history, drugs taken by household members

Pertinent negative physical findings

Product formulation. For manufactured herbal products, whether the product was standardised for which constituent(s) and concentration(s), and for extracts, the

drug-extract ratio

Serum or other fluid drug concentrations. Restart dates

Patient adherence

Last dose-resolution interva

Start and stop doses of concomitant therapies 


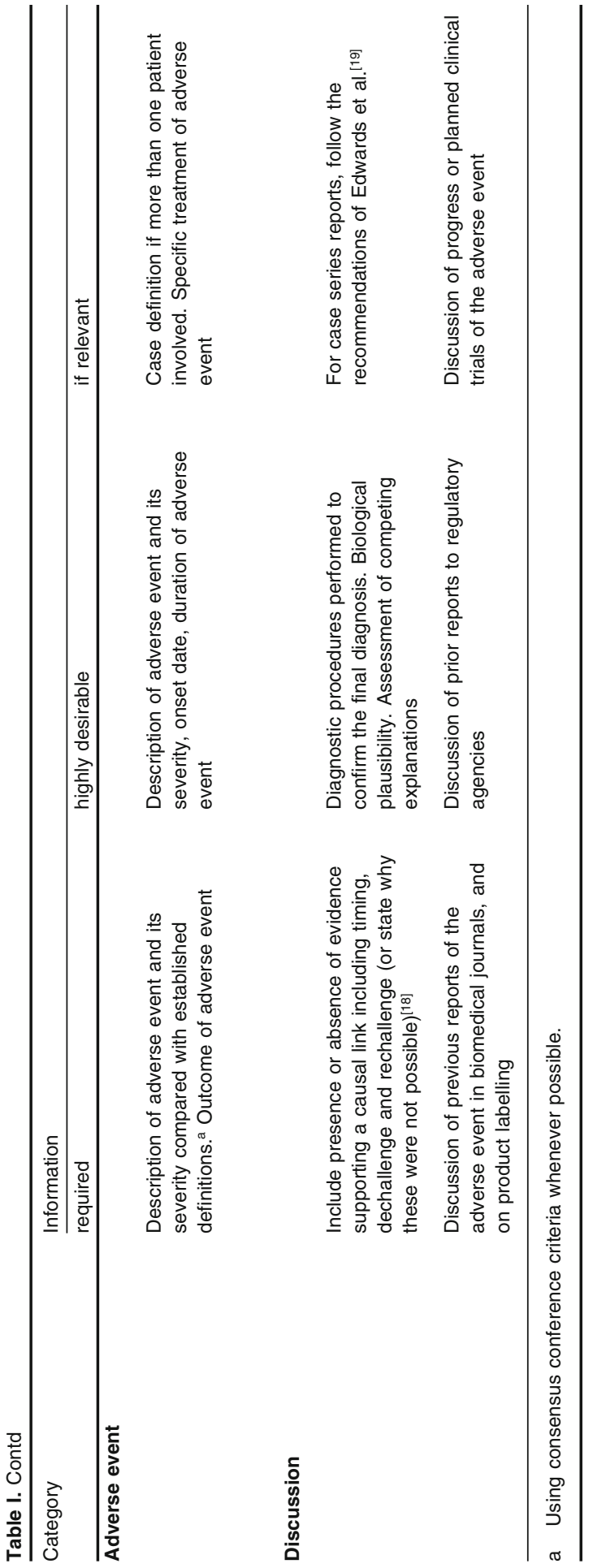

\section{Case Example}

The case example in table II is fictitious and in no way implies causality to any named drug.

\section{Discussion}

The guidelines presented in this paper describe three tiers of key information about suspected adverse events that potential authors of case reports should consider when explaining their case. To the extent possible, inclusion of the required and desirable information will promote a clearer, more structured differential diagnosis for the event. These guidelines build on the original guidelines for publications of suspected adverse drug reactions published in the Drug Information Journal in 1985. ${ }^{[1]}$

Published reports that conform to the guidelines presented here can serve three main purposes. First, well documented adverse event reports can alert practitioners to the possibility of a suspected medical product risk associated event and increase their awareness of it. This heightened sensitivity may allow earlier diagnoses in subsequent cases, with better prognoses through earlier therapy, potentially including suspension of the suspect medication.

Second, for regulators and clinicians in the pharmaceutical and medical product industries who must carefully evaluate adverse event reports, robust and complete details as outlined in the guidelines are invaluable to help identify possible risk factors and differential diagnoses of an adverse event are invaluable. Rare events can also contribute to developing a case definition for an epidemiological study.

Third, the guideline framework can contribute to clinical teaching about assessing suspected adverse events. The framework highlights the need for clinicians to include a possible drug effect in their differential diagnosis of any new medical event, and it outlines the important elements to consider when evaluating a suspected adverse reaction to a drug or medical product.

The failure to develop well documented information in published cases can potentially have negative outcomes. When a publication of a suspected adverse event cites an association of an event with a 
Table II. Case example

BACKGROUND: Published adverse event reports often lack adequate information to evaluate causality. Guidelines have therefore been developed to improve relevant case information content.

OBJECTIVE: To show an example of how the guidelines might be applied to a clinical case report that might be submitted for publication.

CASE: A 75-year-old White man consulted his practitioner for increasing tiredness and right abdominal heaviness over the preceding week. He had a long history of osteoarthritis (OA), was treated with ibuprofen (one to two $200 \mathrm{mg}$ tablets daily) or paracetamol (acetaminophen) as needed (maximum one to two $500 \mathrm{mg}$ tablets daily), and was prescribed diclofenac $75 \mathrm{mg}$ twice daily when his pain was more intense. About 4 weeks before presentation, he was prescribed the recently approved NSAID drug X [brand name $\left.{ }^{\circledR}\right]$ xxxmg twice daily for worsening of $\mathrm{OA}$, and he has used it regularly since. His history included hypertension and hyperlipidemia treated uneventfully with ramipril and simvastatin for several years. His weekly alcohol intake was 5-6 glasses of wine; he had no family history of liver disease, had not travelled recently, and was sexually stable with no history of drug abuse. On presentation, his clinical examination was unremarkable without fever or other signs of infection or inflammation. Laboratory investigations revealed the following levels: ALT $780 \mathrm{U} / \mathrm{L}$ (10 times the upper limit of normal $[\times \mathrm{ULN}])$, AST $792 \mathrm{U} / \mathrm{L}(10 \times \mathrm{ULN})$ and alkaline phosphatase $192 \mathrm{U} / \mathrm{L}(2.5 \times$ ULN). His bilirubin was normal. There was no sign of liver failure or impaired renal function. Liver ultrasound was normal. Titers were negative for hepatitis $B$ and $C$, but markers of previous hepatitis A were noted. A liver biopsy was not performed, and there was no measurement of serum drug concentrations. Drug X was discontinued and his AST and ALT concentrations decreased to about $5 \times$ ULN within 1 week and normalised within 1 month. His alkaline phosphatase level remained slightly raised. No changes were made to any of his other medications. He continued to use OTC analgesics and diclofenac as needed throughout this period.

DISCUSSION: Since a liver biopsy was not undertaken, the available data only allow a broad diagnosis of hepatocellular injury. ${ }^{[20]}$ The demographics, medical history and personal history of this patient did not suggest any underlying hepatic disease. The time sequence of the start of the new drug and onset of the disease is consistent with drug-related hepatocellular injury. ${ }^{[20,21]}$ Although no rechallenge was attempted, the rapid improvement of liver enzyme levels after discontinuation of drug X (i.e. positive dechallenge) suggests an association of the hepatic injury with the use of the drug. The clear regression of liver enzymes despite persistent use makes association with the other drugs used unlikely. Regular use of alcohol and paracetamol might have contributed to the event. There was no indication of current viral hepatitis (negative hepatitis B, C markers, previous hepatitis A) or of infection or gallbladder disease. Other causes of hepatocellular injury (for example, other viruses or toxic exposures) are possible but not suggested by the clinical history. There was no overt alcohol abuse, or use of other known hepatotoxic medications or herbs. A search of Reactions ${ }^{\circledR}$ found no published reports of similar adverse reactions with drug $X$. Although adverse hepatic reactions are not mentioned in the prescribing information for drug $X$, various hepatic reactions have been reported with other drugs in the same therapeutic or chemical classes.

CONCLUSION: Our opinion is that a relationship between the drug and the onset of the apparent hepatitis is plausible, and prescribers and users of drug $X$ should be alert to the possibility of such adverse reactions. This case has been reported to the National Health Authorities (registered as number 06-xxxx) and to the manufacturer of the drug.

particular drug or medical product, it may be overinterpreted by clinicians as a confirmed causal relationship. However, if incomplete, key aspects of the putative association might not be obvious, particularly when the adverse event could also be closely associated with the indication for the drug ('confounding by indication'), or with emerging new symptoms related to the indication ("protopathic bias') or, finally, associated with other concomitant therapy often used with the suspected drug. With inadequate risk information, physicians might avoid prescribing an otherwise useful drug. In addition, multiple poorly documented publications of a single case, either in two journals or as a single case, followed by its inclusion within a case series without proper documentation or referencing, can lead to double counting, which can be problematic with rare events.
Should a case report require the patient's consent? Until recently, consent has not been required, and as reports have always been anonymised, only a few clinicians in the reporting institution would likely recognise the patient. However, with the current strengthening of ethical and privacy guidelines for presenting patient information, consent ideally should now be sought for a detailed presentation of an individual case, since the patient may be recognisable. However, permission might be less important in case series which contain less detail on individual patients. Because patient privacy practices and regulations vary between countries, it may be advisable to consult the relevant data privacy protection board, if one exists, and if in doubt obtain patient consent.

The growing focus on drug safety in the past decade has underscored our lack of knowledge 
about many drug-associated disorders, such as hepatic necrosis and QT abnormalities. Careful descriptions of relevant clinical features in published case reports can contribute to the growth in understanding about the safety of medical products that is essential for prescribing physicians and patients to properly weigh anticipated risks and benefits in their therapeutic choices.

ISPE and ISoP urge biomedical journals and journal editors to adopt these guidelines and encourage schools of medicine, pharmacy, and nursing to incorporate them into their curriculums.

\section{Acknowledgements}

No sources of funding were used in preparing these guidelines and the authors have no conflicts of interest that are directly relevant to the content of these guidelines. This work has been submitted by the International Society of Pharmacoepidemiology (ISPE) and the International Society of Pharmacovigilance (ISoP). ${ }^{1}$ All authors participated as ISPE or ISoP members, and the views expressed do not necessarily represent positions of their government, institution or corporation.

ISPE is dedicated to advancing the health of the public by being an international forum for the open exchange of scientific information among academia, government, and industry and for development of policy; a provider of education; and an advocate for the fields of pharmacoepidemiology and therapeutic risk management. ISoP is an international nonprofit scientific organisation, which aims to foster pharmacovigilance both scientifically and educationally, and improve all aspects of the safe and proper use of medicines, in all countries. ISPE and ISoP hold common symposiums and meetings regularly to further their shared goals. Both societies endorse these guidelines and support their publication.

\section{References}

1. Venulet J. Informativity of adverse reactions data in medical publications. Drug Inf J 1985; 19: 357-65

2. Cohen LG, Rovers JP. Addendum to guidelines for reporting adverse drug reactions. Br Med J (Clin Res Ed) 1988; 296: 1800

3. Aronson JK. Anecdotes as evidence. BMJ 2003; 326: 1346

4. Abanades S, Farre M. Guidelines for anecdotes might include more information. BMJ 2003; 327: 290
5. BMJ paper styles. Drug points [online]. Available from URL: http://bmj.bmjjournals.com/advice/sections.shtml [Accessed 2005 Sep 9]

6. Ferguson JA, Mockbee C, Erbele A, et al. Evaluation of published case reports' standards and notification. Drug Inf J 2002; 36: 303-7

7. DeBakey L, DeBakey S. The case report. I. Guidelines for preparation. Int J Cardiol 1983; 4: 357-64

8. Abrutyn E. Better reporting of adverse drug reactions. Ann Intern Med 1985; 102: 264-5

9. Berneker GC, Ciucci AG, Joyce J. Standards for reporting adverse drug reactions. Br Med J (Clin Res Ed) 1983; 287: 1720

10. Haramburu F, Begaud B, Pere JC, et al. Role of medical journals in adverse drug reaction alerts. Lancet 1985; II: 550-1

11. Venulet J, Blattner R, von Bulow J, et al. How good are articles on adverse drug reactions? Br Med J (Clin Res Ed) 1982; 284: 252-4

12. Kelly WN. The quality of published adverse drug event reports. Ann Pharmacother 2003; 37: 1774-8

13. Loke YK, Price D, Derry S, et al. Case reports of suspected adverse drug reactions: systematic literature survey of followup. BMJ 2006; 332: 335-9

14. Auriche M, Bertrand P, Blay N, et al. Les bonnes pratiques de publication de cas cliniques de pharmacovigilance: commentaires. Groupe de Travail sur les Bonnes Pratiques de Publication de Cliniques en pharmacovigilance: commentaires. Thérapie 1997; 52: 123-7

15. Monteagudo JL. Data exchange in the European pharmacovigilance. Stud Health Technol Inform 1996; 28: 69-77

16. International Conference on Harmonisation; guidance on data elements for transmission of individual case safety reports; availability. Notice. Food and Drug Administration, HHS. Fed Regist 1998; 63: 2396-404

17. Hill AB. The environment and disease: association or causation? Proc R Soc Med 1965; 58: 295-300

18. Naranjo CA, Busto U, Sellers EM, et al. A method for estimating the probability of adverse drug reactions. Clin Pharmacol Ther 1981; 30: 239-45

19. Edwards IR, Lindquist M, Wiholm BE, et al. Quality criteria for early signals of possible adverse drug reactions. Lancet 1990; 336: $156-8$

20. Benichou C. Criteria of drug-induced liver disorders. Report of an international consensus meeting. J Hepatol 1990; 11: 272-6

21. Danan G, Benichou C. Causality assessment of adverse reactions to drugs: I. A novel method based on the conclusions of international consensus meetings: application to drug-induced liver injuries. J Clin Epidemiol 1993; 46: 1323-30

Correspondence: William N. Kelly, William N. Kelly Consulting, Inc., 2147 Warwick Drive, Oldsmar, FL 34677, USA. E-mail: wnkelly@earthlink.net

1 The 'Guidelines for Submitting Adverse Events for Publication', published simultaneously in Pharmacoepidemiology and Drug Safety (2007; 16: 581-587), is a free-access document, without assigned copyright, and may be republished, copied or quoted, without permission, on the condition that its content remains unaltered. Copyright of the exact format in which the 'Guidelines for Submitting Adverse Events for Publication' appear here belongs to Adis Data Information $\mathrm{BV}$, but this version may be copied without permission for non-commercial purposes. 